\title{
1 Anterior eye surface changes following miniscleral contact lens wear
}

\section{INTRODUCTION}

3 The prescription of scleral contact lenses as well as the number of practitioners who fit scleral 4 contact lenses has notably expanded over the last years [Hartan, 2017][Nau, 2017] [Vicent,

5 2018]. Nowadays scleral lens prescription and management is no longer limited to highly 6 specialized care centres [Schornack, 2015]. Significant improvements in visual acuity, vision-

7 related quality of life and ocular surface integrity have been repeatedly reported as a consequence 8 of scleral contact lens wear in cases of corneal ectasia and ocular surface disease [Schornack, 9 2014][Visser, 2007][Lee, 2013][Arumugam, 2014][Ortenberg, 2013][Koppen, 2018].

10 Additionally, scleral lenses are increasingly being considered for refractive error correction even

11 in non-compromised eyes [Schornack, 2015].

12 The interaction between the contact lens and the ocular surface is a crucial factor in assuring the 13 safety and the comfort of the contact lens wear [Jones, 2013][Fadel, 2018]. However, information 14 on how the entire topography of the ocular surface is affected by scleral contact lens wear is 15 scarce. The effect of scleral contact lens wear on corneal shape has been traditionally evaluated 16 with Scheimpflug cameras [Vincent, 2014][Soeters, 2015][Vincent, 2016]. The main limitation 17 of these techniques is that their range of measurement is restricted to the cornea. Anterior 18 segment Optical Coherence Tomography (OCT) allows to expand the imaging range to the 19 corneo-scleral transition and sclera, but the analysis is limited to selected meridians. [Alonso-

20 Caneiro, 2016]

21 Corneo-scleral profilometry has recently proven to be an accurate technique to measure the 22 cornea and the sclera simultaneously in 3-dimensions (3D) $360^{\circ}$ around, in a non-contact way 
23 [Iskander, 2016]. Using this technology, we investigated in a previous work that the ocular

24 surface is altered by short term soft contact lens wear [Consejo CL, 2017]. Scleral lenses are hard

25 and larger than soft lenses, they rest entirely on the sclera, without touching the cornea.

26 Consequently, due to the rigid material, larger size and bearing zone we expect to observe greater

27 changes in ocular surface topography as a consequence of short-term miniscleral contact lens

28 wear than that observed when analysing short-term soft contact lens wear.

29 The aim of this work is to describe and quantify how much the whole anterior eye surface is

30 affected by short-term miniscleral contact lens wear. Alterations in corneal region, corneoscleral

31 junction and sclera up to $16 \mathrm{~mm}$ diameter are considered in this study.

\section{METHODS}

34 This study was approved by the Antwerp University Hospital Research Ethics Committee and

35 adhered to the tenets of the Declaration of Helsinki. All subjects gave written informed consent to

36 participate after the nature and possible consequences of the study were explained. Participants in

37 this study included 12 young, healthy adult subjects (10 females, 2 males) aged $29.9 \pm 5.7$ years

38 old (mean \pm SD). This sample size was chosen based on calculations conducted using previous

39 published data on: scleral topography [Consejo EVER, 2017] and also corneal flattening and

40 morphological scleral changes following short-term contact lens wear [Vicent, 2014] [Alonso-

41 Caneiro, 2014]. The later data suggested that a sample size between 6 and 11 participants would

42 yield $80 \%$ power to detect $30 \mu \mathrm{m}$ morphological changes as a consequence of miniscleral

43 contact lens wear, while the previous published data on scleral topography, [Consejo EVER,

44 2017], suggested that a sample size of 10 participants would yield $80 \%$ power to detect $40 \mu \mathrm{m}$ 
45 differences in scleral elevation at the 0.05 significant level. This value was chosen according to

46 the inherent noise of the measuring device in the corneo-scleral peripheral area. The utilized 47 corneo-scleral topographer was proved to provide below $40 \mu \mathrm{m}$ error for an extended measurement area of $16 \mathrm{~mm}$ diameter in calibrated artificial surfaces.[Iskander, 2016] Prior to

49 commencement of the study, all subjects were screened to exclude those with any

50 contraindications to contact lens wear (i.e., significant tear film or anterior segment

51 abnormalities). All the participants but two were contact lens neophytes. Those two participants

52 were occasional soft contact lens wearers, but discontinued lens wear for 24 hours prior to

53 commencing the study, to minimize the effects of soft lens wear on the ocular surface. None of

54 the subjects were previous rigid contact lens wearers. Participants had no prior history of eye 55 injury, surgery or current use of topical ocular medications, specified by the participants as a part 56 of a background questionnaire.

\section{Contact lens fitting}

58 Contact lens fitting was performed by an experienced optometrist (MVH). The lens designed 59 used was the miniMISA miniscleral lens, provided by Microlens (Arnhem, The Netherlands). 60 The lenses were made of highly gas-permeable materials $(\mathrm{Dk}=\ldots$,,$\ldots$ m central thickness, 61 and had a diameter of $16.5 \mathrm{~mm}$. The lens was inserted into the patients left eye with preservative 62 free saline and assessed using a slit lamp. If regions of corneal bearing were observed, the sagittal 63 depth of the lens was increased (in $100 \mu \mathrm{m}$ increments) and the fit reassessed. Corneal clearance 64 was assessed immediately after lens insertion and 2 hours after lens settling, [Vicent, 2017] using 65 an anterior spectral domain OCT (RTVue, Optovue Inc., Fremont, CA, USA). The callipers 66 within the analysis software were used to determine the position of the back surface of the 67 miniscleral contact lens and the anterior surface of the cornea to provide a measure of the central 
68 corneal clearance at the position of the corneal reflex. The mean initial central corneal clearance

69 was $276 \pm 26 \mu \mathrm{m}$, that was reduced to $225 \pm 23 \mu \mathrm{m}$ after lens settling following 2 hours of 70 miniscleral lens wear.

\section{Data collection}

72 The study was conducted over three sessions on the same day. Each session included six

73 measurements from each eye with a corneo-scleral profilometer (Eye Surface Profiler (ESP),

74 Eaglet Eye BV, Netherlands), a height profilometer with the potential to measure the corneo-

75 scleral topography far beyond the limbus. To determine surface heights, algorithms used in ESP

76 achieve similar levels of accuracy to those reached in keratoscopy based instruments such as

77 Placido disk videokeratoscopes [Iskander, 2016]. Accurate measurements of anterior eye surface using ESP require instillation of fluorescein with a more viscous solution than saline [Iskander, 2016]. The BioGlo (HUB Pharmaceuticals) ophthalmic strips were used to gently touch the upper temporal ocular surface. They were impregnated with $1 \mathrm{mg}$ of fluorescein sodium ophthalmic

81 moisten with one drop of an eye lubricant (HYLO-Parin, $1 \mathrm{mg} / \mathrm{ml}$ of sodium hyaluronate).

82 Subjects were instructed to open their eyes wide prior the measurements with ESP to insure full 83 coverage of the corneo-scleral area. Measurements in which the corneo-scleral area was covered by eyelids were excluded.

85 Baseline measurements were conducted in the morning with a minimum of two hours after

86 awakening in order to control the influence of diurnal variation [Read, 2005] and before contact lenses insertion (0h, session 1, baseline measurements (MB)). Measurements were also acquired immediately post lens removal following 5 hours of wear (session 2, M5) and 3 hours after lens removal (i.e., 8 hours after initial lens insertion) (session 3, M8). Following lens removal the corresponding eye was re-examined using a slit-lamp to assess the anterior eye. Participants were 
91 continuing their normal daily activities between the measurement sessions that constituted

92 office/computer work.

\section{Data analysis}

94 Following data acquisition, the raw anterior eye height data (three columns with $X, Y$, and $Z$ 95 coordinates) was exported from ESP for further analysis. To ensure that the data is not tilted, the 96 realignment was performed by first calculating a geodesic (straight line that joins two points in a 97 given surface) of specific distance from the apex, fitting a 3D plane to the geodesic, and then 98 correcting the data with the estimated tilt. This correction is necessary to ensure the repeatable 99 demarcation of the corneo-scleral region within different measurements.

100 First, limbal transition was calculated in 360 semi-meridians, using a custom written algorithm,

101 as the point corresponding to a certain amount of change in the curvature between cornea and 102 sclera [Consejo, 2016]. Further, a best-fit-circle was estimated using the points which demarcated 103 the anterior limbus surface in each semi-meridian. The planar radius of this circle was termed the 104 planar corneo-scleral limbal radius, or shortly the limbal radius.

105 Secondly, for each 3D map, the sclera and cornea were automatically separated at the level of the

106 limbus, with a certain margin of tolerance, based on the results obtained when calculating limbal

107 radius. Mean elevation of corneal (0.0-11.0 $\mathrm{mm}$ diameter) and scleral region (13.0-16.0 mm 108 diameter) was calculated with custom made software. Scleral annulus was further divided into 109 four sectors for statistical analysis: superior $[50,130]^{\circ}$, inferior $[230,310]^{\circ}$, nasal $[40,320]^{\circ}$ and 110 temporal $[140,220]^{\circ}$. Right eyes were corrected for mirror symmetry.

111 The statistical analysis was performed using SPSS software for Windows version 24.0 (SPSS

112 Inc., Chicago, Illinois, United States). The Shapiro-Wilk test was used to test the distribution type 
113 (Gaussian or non-Gaussian) of all continuous variables. Normality of all sets of data was not

114 rejected ( $\mathrm{p}>0.05)$. The ANOVA-repeated-measurements test (adjustment for multiple

115 comparisons: Bonferroni) was performed to ascertain whether there was a change in limbal radius

116 between sessions. The same test was performed to assess whether there was a change in the mean

117 corneal and scleral elevation between sessions. Mauchly's test of sphericity indicated that the

118 assumption of sphericity had not been violated in any ANOVA case under analysis. The level of

119 significance was set to 0.05 .

\section{$121 \quad$ RESULTS}

122 All data reported in this section are given for correctly fitted miniscleral contact lenses that did

123 not induce any noticeable physiological signs in slit lamp examination. It was found that

124 miniscleral contact lens short-term wear had a statistically significant effect on the corneo-scleral

125 area. In particular, values in limbal radius (Table 1) and scleral elevation after miniscleral lens

126 wear were found to be statistically significant different from baseline records.

Table 1. Limbal radius comparison intra session under the influence of wearing miniscleral contact lens during 5

128 hours period (first column) and without wearing contact lenses, fellow eye (second column). Baseline measurements

129 were acquired in the early morning (MB); immediately after contact lens removal (M5) and three hours after removal

130 (M8); in the same time interval measurements data was acquired for the fellow eye. Data was obtained with ESP and

131 processed with a custom made algorithm. [Consejo, 2016] 'n/a' stands for 'non applicable'.

\begin{tabular}{|c|c|c|c|}
\hline & & $\begin{array}{l}\text { Limbal radius under the } \\
\text { influence of } 5 \text { hour } \\
\text { miniscleral contact lens } \\
\text { wear }\end{array}$ & $\begin{array}{l}\text { Diurnal changes in limbal } \\
\text { radius - no contact lens } \\
\text { wear (fellow eye) }\end{array}$ \\
\hline \multirow{3}{*}{ Mean $\pm \mathrm{SD}(\mathrm{mm})$} & MB & $6.03 \pm 0.16$ & $6.03 \pm 0.14$ \\
\hline & M5 & $6.18 \pm 0.12$ & $6.03 \pm 0.14$ \\
\hline & M8 & $6.08 \pm 0.15$ & $6.02 \pm 0.12$ \\
\hline \multirow{3}{*}{$\begin{array}{l}\text { Testing the difference in limbal radius } \\
\text { between sessions }\end{array}$} & MB vs M5 & $p=0.004$ & $p=0.626$ \\
\hline & MB vs M8 & $p=0.153$ & $p=0.310$ \\
\hline & M5 vs M8 & $p=0.026$ & $p=1.000$ \\
\hline \multicolumn{2}{|c|}{ Average increment $(\mu \mathrm{m})$ (between MB \& M5) } & $146 \pm 80$ & $\mathrm{n} / \mathrm{a}$ \\
\hline \multirow{2}{*}{\multicolumn{2}{|c|}{ Maximum absolute change $(\mu \mathrm{m})$ (between MB \& M5) }} & 340 & $\mathrm{n} / \mathrm{a}$ \\
\hline & & 20 & $\mathrm{n} / \mathrm{a}$ \\
\hline
\end{tabular}


133 The observed increment in limbal radius was reversed 3 hours after contact lens removal for $42 \%$

134 of the participants ( 5 out of 12). It was assumed that limbal radius comes back to its original size

135 when it is within the range of $\pm 20 \mu \mathrm{m}$ from the baseline measurement. That range was chosen

136 according to the lateral resolution of the instrument. The mean difference between M8 and MB

137 limbal radius amounted to $50 \pm 60 \mu \mathrm{m}$. Figure 1 shows the observed increment in limbal radius

138 for the 12 subjects and compares the results with the fellow eye.

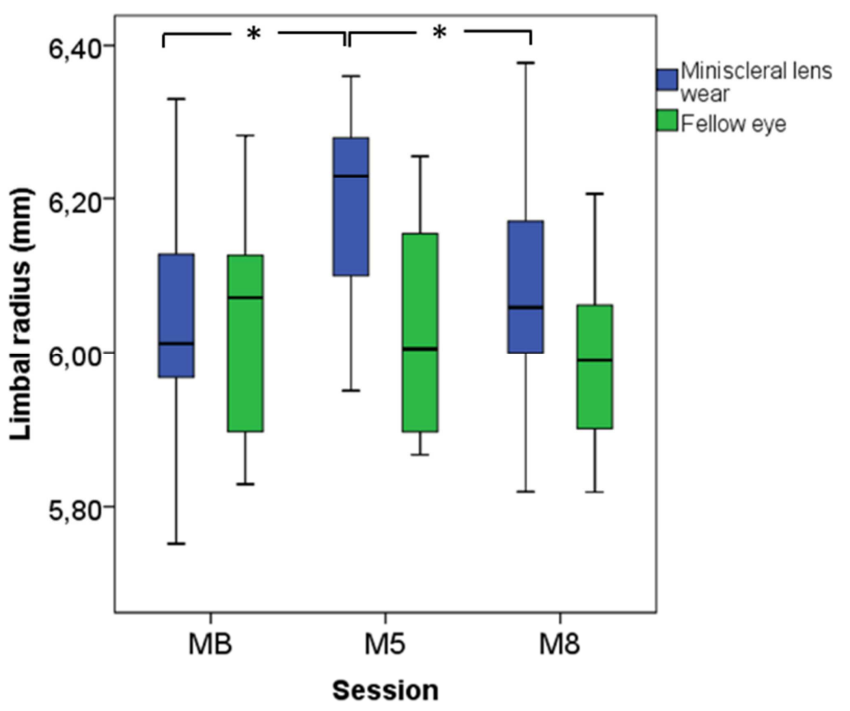

140 Figure 1. The boxplot illustrates the changes in limbal radius for 12 subjects who participated in the experiment,

141 within the three sessions: before contact lens wear (MB), immediately after contact lens removal (M5) and 3 hours

142 after contact lens removal (M8). Blue color corresponds to the eye which wore a miniscleral contact lens, while

143 green color corresponds to the fellow eye. Asterisks denote statistically significant difference between sessions. For

144 details see text.

145 Miniscleral lens wear did not result in significant corneal flattening. The group change over a

$14611.0 \mathrm{~mm}$ corneal diameter was $-3 \pm 17 \mu \mathrm{m}$ immediately after lens removal $(\mathrm{p}=1.000)$, which

147 coincides with the results obtained for the fellow eye between M5 and MB, $-4 \pm 11 \mu \mathrm{m}$

148 ( $\mathrm{p}=0.153)$. Contrarily, miniscleral lens wear resulted in significant scleral flattening. The group 
149 change over the scleral region under analysis (13.0-16.0 mm diameter) amounted to $-122 \pm 90$

$150 \mu \mathrm{m}(\mathrm{p}=0.003)$, which did not completely regressed to baseline values 3 hours after lens removal,

$151-94 \pm 108 \mu \mathrm{m}(\mathrm{p}=0.045)$. Differences within scleral sectors were also found (Figure 2). Within

152 sessions two-way ANOVA test revealed differences between inferior and nasal $(\mathrm{p}<<0.001)$,

153 inferior and superior ( $\mathrm{p}=0.021)$ and nasal and temporal $(\mathrm{p}=0.001)$ sectors.

154

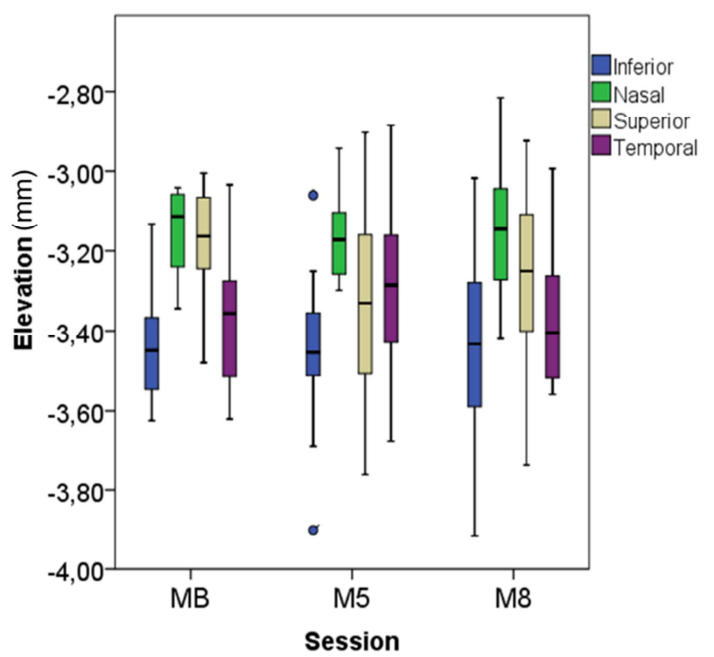

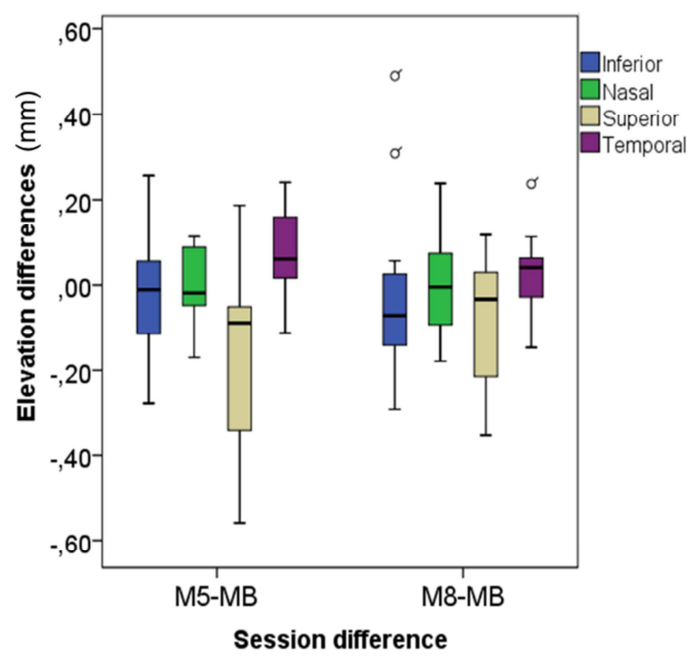

155 Figure 2. Left: Scleral elevation within sectors for each session. Right: Difference respect to baseline in scleral 156 elevation within sectors. Sessions: Before contact lens wear (MB), immediately after contact lens removal (M5) and 1573 hours after contact lens removal (M8).

158 A positive statistically significant correlation was found $\left(R^{2}=0.567, p=0.004\right)$ between scleral 159 flattening (in absolute value) and limbal radius increment, suggesting that as a consequence of 160 miniscleral lens wear, the more the sclera flattens the more limbal radius increments.

161 Statistical power post-hoc estimation was made. The analysis was conducted for $80 \%$ power at 162 the 5\% alpha level. For a sample size of 12 subjects, differences in limbal radius of $60 \mu \mathrm{m}$ and 163 differences in scleral flattening of $40 \mu \mathrm{m}$ could be differentiated. 
166 To our knowledge, this is the first study to examine changes, and their recovery, in corneal, 167 corneo-scleral and scleral topography following short-term miniscleral contact lens wear, 168 analysing $3 \mathrm{D}$ anterior eye surface maps, $360^{\circ}$ around. In this study it was found that a relatively 169 short-term of miniscleral contact lens wear modifies the shape of the anterior eye surface. In 170 particular, limbal radius increment and scleral flattening were observed as a consequence of 171 miniscleral contact lens wear.

172 Limbal radius increment after miniscleral contact lens wear amounted, on average, to $146 \pm 80$ $173 \mu \mathrm{m}$. This value is slightly larger than that obtained when following the same protocol using 174 silicone hydrogel soft contact lenses, $130 \pm 74 \mu \mathrm{m}$. [Consejo CL, 2017]. The observed increment 175 in limbal radius was reversed 3 hours after contact lens removal for $42 \%$ participants when 176 wearing miniscleral contact lenses, and $68 \%$ participants when wearing soft contact lenses. This 177 result is in accordance with the previously reported observation that the more the limbal radius 178 increments, the longer it takes for the effect to be reversed [Consejo CL, 2017].

179 The magnitude of corneal flattening as a consequence of miniscleral contact lens wear, which 180 amounted, on average, to $-3 \pm 17 \mu \mathrm{m}$ was smaller than that previously reported by Vincent and 181 colleagues which amounted, on average, to $-30 \pm 20 \mu \mathrm{m}$ [Vincent, 2014], who found statistical 182 significant differences in corneal shape following miniscleral lens wear. They analysed corneal 183 flattening by means of corneal axial curvature using scheimpflug imaging, while in this work we 184 examined height elevation maps using profilometry. This methodological differences could 185 justify the differences found. In addition, in their work they took into account the diurnal 186 fluctuations in all corneal parameters measured. However, we did not find a statistical significant 187 difference between the control eye and the eye in which the lens was worn. Our findings are in 
accordance with a more recent work, also by Vicent and colleagues [Vicent, 2016], in which

189 posterior corneal curvature was reported to remain stable following 8 hours of miniscleral contact

190 lens wear.

191 Scleral flattening was found to be the most noticeable effect as a consequence of contact lens

192 wear. This flattening amounted, on average, to $-122 \pm 90 \mu \mathrm{m}(\mathrm{p}=0.003)$, implying a $3.7 \pm 2.7 \%$

193 change from its original (baseline) size. This flattening was reduced 3 hour after lens removal, it 194 amounted, on average, to $-94 \pm 108 \mu \mathrm{m}$. However, it was still significantly significant different 195 from baseline values. In a previous work, Alonso-Caneiro and colleagues investigated scleral 196 thickness following 3 hours miniscleral contact lens wear using OCT. [Alonso-Caneiro, 2016] 197 They reported a mean decrease in thickness of $-24 \pm 4 \mu \mathrm{m}$, which diminished 3 hours after lens 198 removal, but was still significantly thinner relative to baseline. Our findings on scleral 199 topography change as a consequence of 5 hours of miniscleral lens wear are in line with their 200 results.

201 Scleral flattening as a consequence of miniscleral lens wear was not uniformly distributed $360^{\circ}$ 202 around. Statistical significant differences in scleral flattening were found among sectors, being 203 the superior sector the most affected by miniscleral lens wear (Figure 2). Scleral toricity [Consejo 204 EVER, 2017][Ritzmann, 2017][Bandlitz, 2017] may result in an uneven distribution of the load 205 for a spherical lens design, like the ones used on this experiment, which might consequently 206 contribute to uneven compression across quadrants. Orientation of extraocular rectus muscle 207 insertions, eye lid forces and lid position have been designated as potential factors influencing 208 scleral shape [Ritzmann, 2017]. Likely, these factors would also influence the effect of wearing 209 miniscleral contact lenses. In a recent work on limbal shape differences in radial distance among 210 quadrants were found, being the superior semimeridian the shortest.[Consejo JCRS, 2017] This 
211 difference was justified by the effect of the eyelid pressure on this area. We conjecture that,

212 precisely due to the eye lid forces and position, [Read, 2006][Read, 2007] the greatest changes in

213 scleral shape as a consequence of miniscleral contact lens wear was observed in the superior

214 sector.

215 The interaction of scleral contact lenses with the anterior eye surface and the influence they have

216 on the physiological processes of corneal tissue is fundamental to ensure a safe wear. Static

217 theoretical models, that did not account for dynamic tear changes that occur during lens wear,

218 have been proposed regarding the oxygen supply to the cornea during scleral lens wear.

219 [Michaud, 2012] [Compan, 2014][Jaynes, 2015]. Based on theoretical calculations, it was

220 suggested that the higher the Dk value, the better in terms of minimizing hypoxia-induced corneal

221 swelling as a consequence of contact lens wear. Recently, a number of short-term clinical studies

222 have attempted to quantify corneal hypoxic changes as a result of scleral lens wear (high Dk).

223 [Vincent, 2014][Compan, 2014][Frisani, 2015]. The results from these works suggested that

224 modern high Dk miniscleral contact lenses, do not induce clinically significant corneal edema

225 following short term of scleral lens wear. Miniscleral contact lenses used during this study met

226 this requirement. However, different reaction and corneal response to contact lens wear

227 depending on the distance from its center could be expected because contact lens thickness is

228 usually not uniform.

229 Note that the results presented need to be put in perspective by considering the instrument's 230 measurement noise. The ESP corneo-scleral topographer used for data acquisition has been

231 demonstrated to provide an RMS error of $<10 \mu \mathrm{m}$ for the central $8 \mathrm{~mm}$ area of a calibrated 232 artificial surface and $<40 \mu \mathrm{m}$ for an extended measurement area of $16 \mathrm{~mm}$.[Iskander, 2016] Our 
233 analysis was performed for an area with a diameter of $0-16 \mathrm{~mm}$. It is worth noting that the

234 internal measurement error of the device is minor in comparison to the values reported.

235 The small sample size could be seen as a limitation of the study, besides the prior power analysis, 236 confirmed by the post hoc test. All participants were young and healthy with normal cornea and 237 sclera, and no history of ocular disease or scleral lens wear. Consequently, the results must be 238 interpreted with caution and may not be applicable for older patients or those with ocular disease 239 or abnormal anterior eye surface.

240 The market on scleral lenses has tremendously increased over the last years. Advances in 241 ophthalmic instrumentation played a key role in that expansion. Also, the rise of formal pathways 242 to undertake training to expand clinical capabilities related to contact lens fitting not only for 243 qualified practitioners but also at undergraduate level [Vicent, 2018] has facilitated that more 244 practitioners included scleral lenses in their portfolio. Similarly, the expanding governmental 245 regulations that might limit the use of trial lenses in clinical practice, [Lian, 2017] is another 246 reason that has contributed to the expansion of scleral lenses. The asymmetrical nature of the 247 sclera or limbal bearing have been acknowledged as fitting challenges associated with scleral 248 contact lenses [Walker, 2016]. Limbal and scleral shape play a fundamental role on scleral lens 249 design [Fadel, 2018]. Consequently, gaining knowledge on how the physiology of these 250 structures is affected by scleral lens wear might help practitioners in the fitting process and 251 follow up.

252 In the present study, limbal radius increment was found for all, except for two, participants. For 253 those two subjects the limbal radius increment of $20 \mu \mathrm{m}$ was at the resolution limit of the 254 instrument. In addition, those two participants were the only ones that did not experience a 255 significant scleral flattening as a consequence of miniscleral contact lens wear. These results 
remark the importance of lens-subject biocompatibility. It is important to notice that in some of

257 the studied cases more than $0.6 \mathrm{~mm}$ increment in limbal diameter or $300 \mu \mathrm{m}$ scleral flattening were found and these changes were unavailable to the examiner using a slit lamp.

261 Short-term miniscleral contact lens wear alters corneo-scleral and scleral topography but do not 262 produce significant corneal shape changes. Gaining knowledge on the effects of lens settling, could help the practitioner prevent cases of scleral blanching or discomfort due to an excessive compression of the lens.

\section{REFERENCES}

267 Alonso-Caneiro D, Vincent SJ, Collins MJ. 2016. Morphological changes in the conjunctiva, episclera and 268 sclera following short-term miniscleral contact lens wear in rigid lens neophytes. Contact Lens and 269 Anterior Eye 39(1):53-61.

270 Arumugam AO, Rajan R, Subramanian M, Mahadevan R. 2014. PROSE for irregular corneas at a tertiary 271 eye care center. Eye Contact Lens 40(2):71-3.

272 Bandlitz S, Baumer J, Conrad U, Wolffsohn J. 2017. Scleral topography analysed by optical coherence 273 tomography. Cont Lens Anterior Eye 40(4):242-7.

274 Bleshoy H and Pullum KW. 1988. Corneal response to gas-permeable impression scleral lenses. Journal of 275 the British Contact Lens Association 11(2):31-4.

276 Compan V, Oliveira C, Aguilella-Arzo M, Mollá S, Peixoto-de-Matos SC, González-Méijome JM. 2014. 277 Oxygen diffusion and edema with modern scleral rigid gas permeable contact lenses. Invest Ophthalmol 278 Vis Sci 55(10):6421-9.

279 Consejo A, Iskander R, Rozema J. 2017. Mean shape of the human sclera. Acta Ophthalmol 95(S259).

280 Consejo A and Iskander DR. 2016. Corneo-scleral limbus demarcation from 3D height data. Contact Lens 281 and Anterior Eye .

282 Consejo A, Bartuzel MM, Iskander DR. 2017. Corneo-scleral limbal changes following short-term soft 283 contact lens wear. Cont Lens Anterior Eye 40(5):293-300.

284 Fadel D. 2018. The influence of limbal and scleral shape on scleral lens design. Contact Lens and Anterior 285 Eye .

286 Frisani M, Beltramo I, Grec M. 2015. Changes in corneal thickness by miniscleral contact lenses. Contact 
Harthan J, Nau CB, Barr J, Nau A, Shorter E, Chimato NT, Hodge DO, Schornack MM. 2017. Scleral lens prescription and management practices: The SCOPE study. Eye Contact Lens .

290 Iskander DR, Wachel P, Simpson PN, Consejo A, Jesus DA. 2016. Principles of operation, accuracy and precision of an eye surface profiler. Ophthalmic and Physiological Optics 36(3):266-78.

Jaynes JM, Edrington TB, Weissman BA. 2015. Predicting scleral GP lens entrapped tear layer oxygen tensions. Cont Lens Anterior Eye 38(1):44-7.

294 Jones L, Brennan NA, González-Méijome J, Lally J, Maldonado-Codina C, Schmidt TA, Subbaraman L, Young G, Nichols JJ. 2013. The TFOS international workshop on contact lens discomfort: Report of the contact lens materials, design, and care subcommitteeTFOS international workshop on CLD. Invest Ophthalmol Vis Sci 54(11):TFOS37-70.

Koppen C, Kreps EO, Anthonissen L, Van Hoey M, Dhubhghaill SN, Vermeulen L. 2018. Scleral lenses reduce the need for corneal transplants in severe keratoconus. Am J Ophthalmol 185:43-7.

300 Lee JC, Chiu GB, Bach D, Bababeygy SR, Irvine J, Heur M. 2013. Functional and visual improvement with prosthetic replacement of the ocular surface ecosystem scleral lenses for irregular corneas. Cornea 32(12):1540-3.

303 Lian K, Napper G, Stapleton FJ, Kiely PM. 2017. Infection control guidelines for optometrists 2016.

304 Clinical and Experimental Optometry 100(4):341-56.

305 Nau CB, Harthan J, Shorter E, Barr J, Nau A, Chimato NT, Hodge DO, Schornack MM. 2017. 306 Demographic characteristics and prescribing patterns of scleral lens fitters: The SCOPE study. Eye 307 Contact Lens .

308 Ortenberg I, Behrman S, Geraisy W, Barequet IS. 2013. Wearing time as a measure of success of scleral lenses for patients with irregular astigmatism. Eye Contact Lens 39(6):381-4.

310 Read SA, Collins MJ, Carney LG. 2007. The influence of eyelid morphology on normal corneal shape. 311 Invest Ophthalmol Vis Sci 48(1):112-9.

312 Read SA, Collins MJ, Carney LG. 2005. The diurnal variation of corneal topography and aberrations. 313 Cornea 24(6):678-87.

314 Read SA, Collins MJ, Carney LG, Iskander DR. 2006. The morphology of the palpebral fissure in 315 different directions of vertical gaze. Optom Vis Sci 83(10):715-22.

316 Ritzmann M, Caroline PJ, Börret R, Korszen E. 2017. An analysis of anterior scleral shape and its role in 317 the design and fitting of scleral contact lenses. Contact Lens and Anterior Eye .

318 Schornack MM. 2015. Scleral lenses: A literature review. Eye \& Contact Lens 41(1):3.

319 Schornack MM, Pyle J, Patel SV. 2014. Scleral lenses in the management of ocular surface disease. 320 Ophthalmology 121(7):1398-405.

321 Soeters N, Visser ES, Imhof SM, Tahzib NG. 2015. Scleral lens influence on corneal curvature and 322 pachymetry in keratoconus patients. Contact Lens and Anterior Eye 38(4):294-7.

323 Vincent SJ. 2018. The Rigid Lens Renaissance: A Surge in Sclerals.

324 Vincent SJ, Alonso-Caneiro D, Collins MJ. 2014. Corneal changes following short-term miniscleral 325 contact lens wear. Contact Lens and Anterior Eye 37(6):461-8.

326 Vincent SJ, Alonso-Caneiro D, Collins MJ. 2017. The temporal dynamics of miniscleral contact lenses: 327 Central corneal clearance and centration. Contact Lens and Anterior Eye .

328 Vincent SJ, Alonso-Caneiro D, Collins MJ, Beanland A, Lam L, Lim CC, Loke A, Nguyen N. 2016. 329 Hypoxic corneal changes following eight hours of scleral contact lens wear. Optom Vis Sci 93(3):293-9. 
330 Visser ES, Visser R, van Lier HJ, Otten HM. 2007. Modern scleral lenses part II: Patient satisfaction. Eye 331 Contact Lens 33(1):21-5.

332 Walker MK, Bergmanson JP, Miller WL, Marsack JD, Johnson LA. 2016. Complications and fitting 333 challenges associated with scleral contact lenses: A review. Cont Lens Anterior Eye 39(2):88-96. 